\title{
Improvement in Inhalation Technique: A Pilot Quality Improvement Project
}

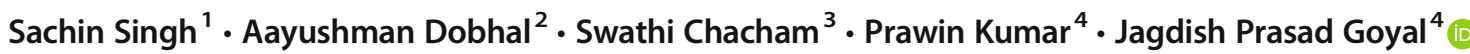

Received: 25 February 2020 / Accepted: 19 June 2020 / Published online: 10 July 2020

(C) Dr. K C Chaudhuri Foundation 2020

To the Editor: Incorrect inhalation technique may be responsible for poor asthma control and increase financial burden as well as poor quality of life to asthma patient [1]. This pilot quality improvement (QI) project was done at a pediatric asthma clinic in AIIMS, Rishikesh for a period of three months. We decided to improve the inhalation technique of patients by using a quality improvement method consisting of 'plan do study act' (PDSA) cycles. The current process of inhalation technique was studied and fishbone analysis was done which revealed the reasons for improper inhalation techniques. Lack of patient education, lack of awareness and motivation of healthcare staff, myths and misconceptions regarding inhalation technique were few reasons for improper inhalation technique. An inhaler specific standardized checklist consisting of eight items designed by the Dutch Lung Foundation [2] was prepared. The children were scored according to the correct steps done in asthma clinic. If a child demonstrated all steps correctly, he or she was given 8 score. Several PDSA cycles were performed after collecting baseline data on consecutive asthma clinics.

The improvement in the inhalation technique was the process measure. This was defined as an increase in the median score of the correct inhalation technique from baseline. The baseline data on inhalation technique were collected. The median score of the inhalation technique was $4, \operatorname{IQR}(4,5)$. After carrying out all PDSA the score improved to 6 , IQR $(6,7)$. This change was sustained on subsequent asthma clinics. Asthma control was also

\section{Jagdish Prasad Goyal}

jpgoyal@rediffmail.com

1 Department of Pediatrics, Post Graduate Institute of Medical Education and Research, Chandigarh, India

2 Department of Biochemistry, All India Institute of Medical Sciences, New Delhi, India

3 Department of Pediatrics, All India Institute of Medical Sciences, Rishikesh, Uttarakhand, India

4 Department of Pediatrics, All India Institute of Medical Sciences, Jodhpur, Rajasthan, India better in children after this QI project. The most common cause for the wrong inhalation technique was not shaking the inhaler followed by checking of movement of the valve of the spacer and keeping chamber in the horizontal position.

The need for asthma education regarding the correct inhalation technique is important for effective asthma management [3]. The lack of monitoring of treatment and inappropriate counselling, in asthma management may lead to poor asthma control; QI methodology may lead to an improvement in asthma care [4, 5].

To conclude, this project highlights that QI (trying several simple changes to improve patient care) is a feasible and lowcost way to improve care for pediatric asthma patients.

Acknowledgments This pilot project was conducted as part of QI training sessions at All India Institute of Medical Sciences, New Delhi under the guidance of teams from IHI and USAID ASSIST. The authors are thankful to Dr Sonali Vaid for her input in the preparation of this manuscript.

\section{Compliance with Ethical Standards}

Conflict of Interest None.

\section{References}

1. Al-Jahdali H, Ahmed A, Al-Harbi A, et al. Improper inhaler technique is associated with poor asthma control and frequent emergency department visits. Allergy Asthma Clin Immunol. 2013;9:8.

2. Kamps AW, van Ewijk B, Roorda RJ, Brand PL. Poor inhalation technique, even after inhalation instructions, in children with asthma. Pediatr Pulmonol. 2000;29:39-42.

3. Boulet L-P. Asthma education: an essential component in asthma management. Eur Respir J. 2015;46:1262-4.

4. Mansour ME, Rose B, Toole K, Luzader CP, Atherton HD. Pursuing perfection: an asthma quality improvement initiative in school-based health centers with community partners. Public Health Rep. 2008;123:717-30.

5. Dolins JC, Powell J, Wise E, et al. Improving asthma care by building state wide quality improvement infrastructure. Pediatrics. 2017;140:e20161612.

Publisher's Note Springer Nature remains neutral with regard to jurisdictional claims in published maps and institutional affiliations. 